\section{Nierensteinaufklärung via YouTube}

\section{Auf das Videoportal YouTube greifen tägliche Millionen von Nutzern zu. Ein internationales Forscherteam hat nun untersucht, ob sich YouTube eignet, über Nierensteinleiden zu informieren oder ob die Inhalte - medizinisch gesehen - eher irreführend sind.}

ndische und amerikanische Forscher durchsuchten fast zweihundert Videos $\mathrm{zu}$ relevanten Informationen im Bezug auf Nierensteine. Inhalte, die über Präventionsmaßnahmen und anerkannte Therapien aufklärten oder bekannte Symptome beschrieben, stuften die Wissenschaftler als nützlich ein. In der Kategorie „irreführend“ landeten Darstellungen über pflanzliche Heilmittel, Diäten mit Bier, Cola oder Fleisch, sowie Heiler, die versprachen, Steinleiden durch Hüpfen oder Heraussaugen zu beseitigen. Eine dritte Gruppe bildeten Videos mit persönlichen Erfahrungen. Die Videos stammten unter anderem von Universitäten, Nachrichtenagenturen und aus Heilwerbespots und von unabhängigen Nutzern.

Fast zwei Drittel (58,3\%) der Videos zeigten nach Meinung der Mediziner hilfreiche Inhalte, während $18,1 \%$ als eher irreführend eingestuft wurden. In mehr als einem Fünftel der Fälle schilderten Betroffene eigene Erfahrungen. Die meisten informativen Inhalte stammten von
YouTube-Nutzern selbst. Aus dieser Quelle kamen aber auch die meisten als Fehlinformation eingestuften Videos. Universitäten oder öffentliche Regierungsstellen veröffentlichten lediglich zehn Videos beziehungsweise gerade mal ein Video.

Die Mediziner werteten nicht nur die Quellen und den Inhalt aus, sondern auch die Anzahl der Zugriffe. Dabei schienen die Nutzer die Nützlichkeit der Videos gut einzuschätzen: Nur 2,8\% der am Thema Interessierten sahen sich fragwürdige Informationen an. Dagegen klickten 47,2\% auf die als nützlich eingestuften Seiten. Fast die Hälfte $(49,9 \%)$ interessierte sich für persönliche Schilderungen Betroffener. Auch die Benotung der Videos von Seiten der Konsumenten zeigte, dass die Internetsurfer Wichtiges von Unwichtigem trennen können: Relevante Informationen wurden signifikant besser bewertet.

Fazit: Vor dem Hintergrund, dass monatlich 50 Millionen Amerikaner Informationen zu Gesundheitsthemen aus dem
Internet beziehen und YouTube als eine frei zugängliche Plattform täglich über 100 Millionen Nutzer anzieht, sahen die Autoren eine Chance für eine glaubwürdige Aufklärung. Auch wenn sie selbstkritisch anmerkten, dass das Studiendesign subjektiv und punktuell sei, wäre das Videoportal eine gute Möglichkeit an Patienten heranzutreten und so ihr Verhalten präventiv zu beeinflussen. Es müssten nur mehr Inhalte aus zuverlässigen Quellen stammen.

lav

Sood, A. et al. You tube as a source of Information in Kidney Stone Disease, Urology 2011; 77: 558-62

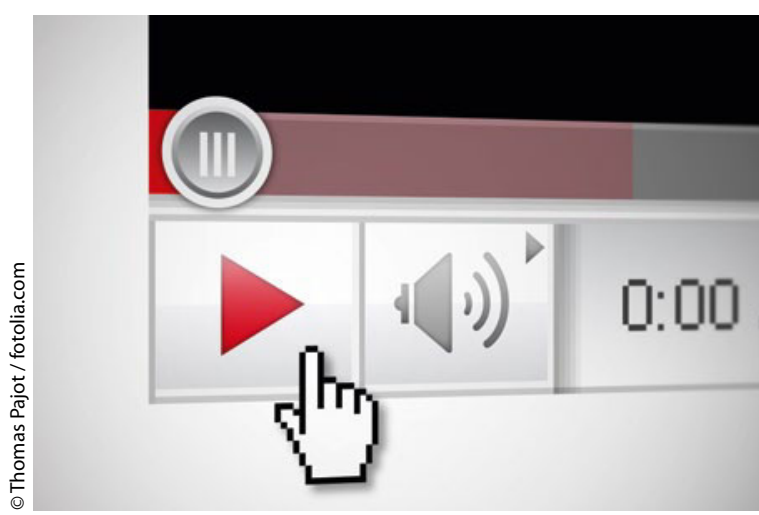

Videos bei YouTube: Eine Kontrolle darüber, welche Informationen nützlich sind und welche eher nicht, gibt es nicht. 